\title{
INVENTÁRIO TAXONÔMICO PRELIMINAR DA COMUNIDADE BENTÔNICA NOS RESERVATÓRIOS DO RIO JAGUARI E DO RIO JACAREÍ - SISTEMA CANTAREIRA SABESP - SÃO PAULO, SP.
}

\author{
Daniele Cristina Schiavone ${ }^{1}$
}

Thiago Enrique Gonçalves Monzane ${ }^{2}$

\author{
José Valdecir de Lucca ${ }^{3}$
}

RESUMO: Os macroinvertebrados bentônicos veem recebendo maior atenção por sua importância nos processos ecológicos, participando do fluxo de energia e ciclagem de nutrientes nos sistemas aquáticos, desenvolvendo um papel importante na troca de fósforo e nitrogênio entre o sedimento e a água de interface. Nas cadeias alimentares são considerados intermediários e ecologicamente importantes, predadores de algas e microrganismos, predados por peixes e outros vertebrados. Os macroinvertebrados bentônicos também são importantes nos estudos de avaliação de impactos ambientais, refletindo o estado de conservação ou degradação do ecossistema. Entre as características que os tornam organismos eficazes nesses estudos destacam-se, o clico de vida longo que explicam os padrões temporais de alterações causadas pelas perturbações, ampla tolerância ao vários graus de poluição e abundância em todos os sistemas aquáticos. O presente estudo objetivou a realizar o levantamento da comunidade de macroinvertebrados bentônicos em dois reservatórios pertencentes ao sistema Cantareira, Jacareí e Jaguari (SP), em agosto de 2012. A coleta foi realizada com uma draga VanVeen, com área amostral de $477 \mathrm{~cm}^{2} \mathrm{em}$

\footnotetext{
${ }^{1}$ Bióloga, Universidade de São Paulo. danischiavone@yahoo.com.br

${ }^{2}$ Biólogo, Universidade Federal de São Carlos. thiagomonzane@gmail.com

${ }^{3}$ Biólogo, Universidade Federal de São Carlos. jvlucca@bol.com.br
} 


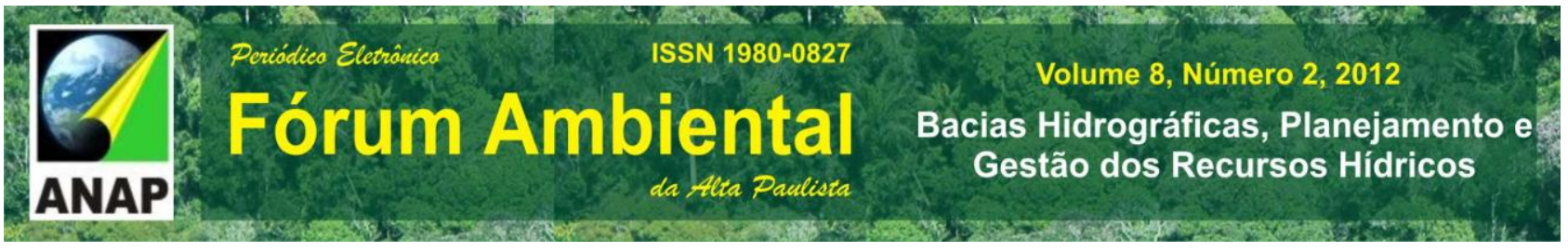

tréplicas, foram feitas medidas "in situ" de dados abióticos e determinação da concentração de fósforo total (PT) em laboratório. A abundância total de indivíduos foi de 709, distribuídos em 9 táxons, onde a família Chironomidae e a Classe Oligochaeta foram as mais representativas numericamente. O Indice de Estado Trófico reveleou um sistema que variou de oligotrófico a eutrófico no Jaguari e de oligotrófico a mesotrófico no Jacareí.

Palavras-chave: Macroinvertebrados bentônicos. Biodiversidade. Sistema Cantareira.

\section{INTRODUÇÃO}

Os macroinvertebrados bentônicos constituem um instrumento adequado para estudos de impactos sobre ambientes aquáticos, pois refletem o estado de conservação ou degradação do ecossistema. Dentre as características que tornam estes organismos eficazes neste tipo de estudo destacam-se: abundância em todos os sistemas aquáticos, baixa mobilidade, ciclo de vida longo, possibilitando a explicação de padrões temporais de alterações causadas por perturbações, ampla variedade de tolerância a vários graus e tipos de poluição e funcionalidade, como integradores das condições ambientais, estando presentes antes e após eventos impactantes (ROSENBERG \& RESH, 1993).

Além disso, os macroinvertebrados bentônicos têm recebido maior atenção devido à sua importância nos processos ecológicos, por meio da participação no fluxo de energia e ciclagem de nutrientes nos sistemas aquáticos, desempenhando papel importante na troca de fósforo e nitrogênio entre o sedimento e a água de interface (GARDNER et al. 1983 apud LUCCA, 2002; FUKUHARA \& SAKAMOTO, 1988 apud LUCCA, 2002). Esses organismos constituem uma comunidade extremamente diversa, tanto taxonomicamente quanto ecologicamente. Entre os principais grupos de macroinvertebrados bentônicos destacam-se as larvas de insetos que são geralmente encontradas em grande quantidade entre elas, os representantes da família Chironomidae são considerados os mais abundantes da comunidade bentônica, (PINDER, 1983. CALLISTO et al., 2002).

O objetivo desse trabalho foi analisar e identificar a composição, a biodiversidade e a densidade da comunidade bentônica, visando identificar a ocorrência de diferenças na diversidade de espécies, relacionando com as características físicas e químicas da água 
em dois reservatórios do sistema Cantareira: reservatórios de Jacareí e Jaquari. Este sistema é responsável pelo abastecimento de água para 8,8 milhões de pessoas sendo os reservatórios de Jacareí e Jaguari responsáveis por $45 \%$ desse total; todavia, a qualidade da água captada tem sofrido grande pressão antrópica nas últimas décadas, principalmente devido à alteração no uso e ocupação de seu entorno, essencialmente rural na década de 70 , atualmente impactado pelo aumento populacional e pela atividade industrial.

\section{OBJETIVOS ESPECÍFICOS}

1. Caracterizar a composição e a densidade da comunidade bentônica, visando identificar a ocorrência de diferenças na diversidade de espécies e na densidade populacional da comunidade bentônica nos dois reservatórios;

2. Identificar a comunidade de macroinvertebrados bentônicos nos reservatórios de Jacareí e Jaguari, contribuindo para o conhecimento da biodiversidade dos sistemas de água doce do estado de São Paulo;

3. Determinar as características físicas e químicas das lagoas selecionadas, ampliando o conhecimento limnológico sobre as mesmas e verificando a possível relação entre as características físicas e químicas da água dos reservatórios com a estrutura da comunidade bentônica;

\section{METODOLOGIA}

A coleta de sedimento para análise da comunidade de macroinvertebrados bentônicos foi realizada em agosto de 2012 em 4 pontos no reservatório de Jacareí

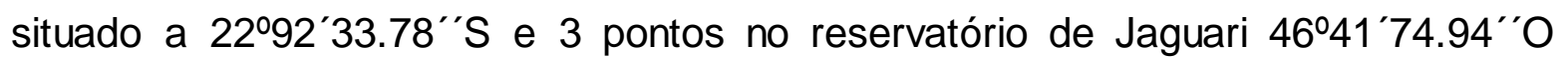
(Figura 1). Utilizou-se uma draga do VanVeen, com $477 \mathrm{~cm}^{2}$ de diâmetro de área amostral, realizando-se 3 dragagens por ponto de coleta. Após coletadas as amostras foram lavadas em peneira com $0,21 \mathrm{~mm}$ de abertura de malha. $O$ material retido na peneira foi preservado em formol $8 \%$ no próprio local de coleta. No 
laboratório, utilizou-se uma solução do corante rosa bengala a 10\% a fim de corar os organismos presentes na amostra e facilitar a visualização dos mesmos. Utilizando-se uma bandeja transiluminada, os organismos bentônicos foram triados e preservados em álcool a 70\%. A identificação foi feita sob estereomicroscópio óptico seguindo a chave de identificação: MERRITT \& CUMMINS (1984); ROLDAN-PÉREZ (1988); EPLES (1992); TRIVINHO-STRIXINO \& STRIXINO (1995), RIGHI (1984) e BRINKHURST \& MARCHESE (1993), entre outras.

Foram realizadas medidas "in situ" do potencial Hidrogeniônico, condutividade elétrica, oxigênio dissolvido e temperatura com equipamentos da marca Horiba modelo U-10. Realizou-se também coletas de água para determinação da concentração de fósforo total (PT) seguindo a metodologia descrita em VALDERRAMA, 1981.

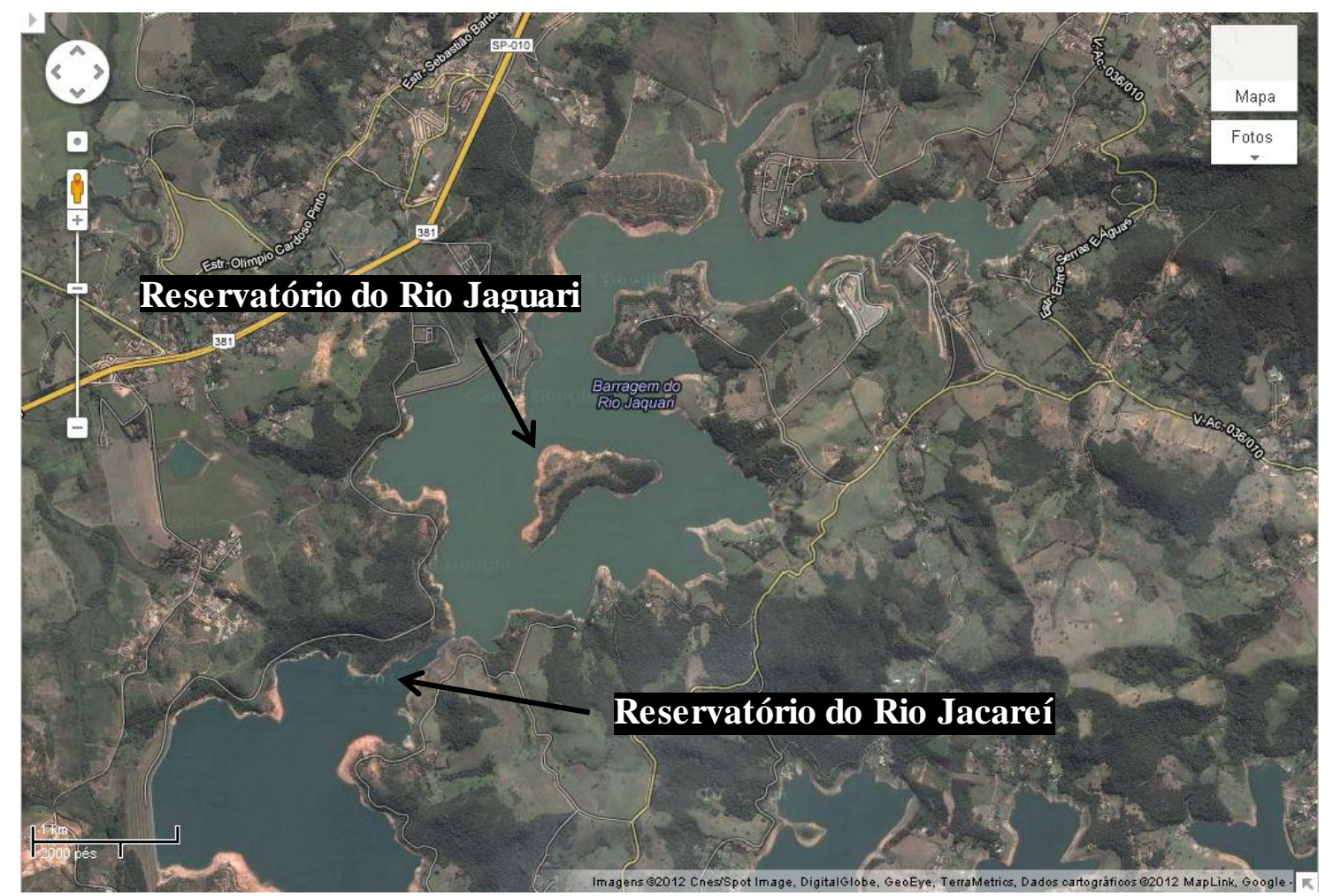

Figura 1. Imagem de satélite dos Reservatórios dos rios Jaguari/Jacareí (ênfase: Reservatório do rio Jaguari; Reservatório do rio Jacareí). Localização: 22ํำ33.78”S / 46ำ1’74.94”O (Fonte: Google Earth, 2012). 


\section{Análise dos Dados:}

a. Foi verificado a presença/ausência e calculado a densidade dos táxons de acordo com a formula proposta por WELCH, 1948).

$$
n=\left(0 / a^{*} s\right)^{*} 10.000
$$

b. A diversidade de espécies de cada ponto amostrado foi calculada através da fórmula:

$$
\begin{gathered}
H^{\prime}=-\Sigma \mathrm{pi}^{*} \log \mathrm{Pl} \\
\mathrm{pi}=\mathrm{ni} / \mathrm{N}
\end{gathered}
$$

onde: ni é o número de indivíduos da espécie numa amostra e $\mathbf{N}$ é o número total de indivíduos presentes na amostra (MAGURRAN, 1988);

c. Índice de Similaridade: Equivalente ao índice DICE, no sentido de se estabelecer o grau de semelhança entre as composições de organismos e as suas respectivas localizações. Este índice baseia-se nos dados de presença e ausência das espécies. O coeficiente de Sorensen (Ss) é expresso por:

$$
\mathrm{Ss}=2 \mathrm{a} / 2 \mathrm{a}+\mathrm{b}+\mathrm{c}
$$

onde: $\mathbf{a}=$ número de espécies comuns a ambas amostras; $\mathbf{b}=$ número de espécies presentes somente na amostra b; c = número de espécies presentes somente na amostra c (SORENSEN, 1948).

Para realizar o agrupamento e construir do dendrograma de similaridade, foi utilizado o programa estatístico Palaeontological Statistics Software Package v. 1,74, PAST (HAMMER et al., 2001).

d. Com os resultados de concentração de fósforo total (PT) foi calculado o índice de estado trófico (IET) através da formula abaixo proposta por TOLEDO JR et al. 1983 modificado para lagos e reservatórios tropicais.

$$
\operatorname{IET}(\mathrm{P})=10 \times\left(6-\frac{\ln (80,32 / \mathrm{P})}{\ln 2}\right)
$$

Os critérios para classificação desse índice são apresentados na tabela 1.

Tabela 1. Critérios de classificação trófica através do IET, modificado por TOLEDO JR et al. (1983). 


\begin{tabular}{cc}
\hline \multicolumn{2}{c}{ Estado Trófico IET - P } \\
\hline Oligotrófico & $<44$ \\
Mesotrófico & $44-54$ \\
Eutrófico & $>54$ \\
\hline
\end{tabular}

\section{RESULTADOS}

Na Tabela 2 são apresentados a composição taxonômica, abundância absoluta e relativa e a densidade numérica (ind. $\mathrm{m}^{2}$ ) dos macroinvertebrados bentônicos no reservatório de Jaguari. No total foram coletados 259 espécimes. A maior densidade numérica foi registrada no ponto JA 01 com 193 ind. $\mathrm{m}^{2}$, destes, 154 ind. $\mathrm{m}^{2}$ pertencem a Classe Oligochaeta. De acordo com BRINKHURST \& JAMIESON (1971), os representantes da Classe Oligochaeta apresentam ampla distribuição geográfica e suas populações podem alcançar grandes densidades. Por possuírem grande plasticidade e capacidade de sobreviver em ambientes eutrofizados, são quase sempre frequentes. Esse fato provavelmente explique a elevada abundância desse grupo no ponto JA 01 que foi classificado como eutrófico (Tabela 4).

No reservatório de Jacareí foram registrados a ocorrência de 8 táxons e um total de 450 ind. $\mathrm{m}^{2}$ (Tabela 3). Entre os táxons, Chironomidae foi notadamente o mais representativo numericamente, contendo no ponto JC 02 (Jacareí) 273 ind. $\mathrm{m}^{2}$. O fato das larvas de Chironomidae terem sido coletadas em todas as estações de amostragem se deve ao fato dos representantes da família Chironomidae serem considerados os mais abundantes na comunidade bentônica (PINDER, 1983; CALLISTO et al., 2002) e normalmente são dominantes nos ecossistemas aquáticos devido à capacidade de tolerar diferentes tipos de condições extremas (CRANSTON, 1995; DI GIOVANNI et al., 1996). Este fato corrobora com os resultados obtidos no reservatório de Jacareí onde os Chironomidae, foram os mais representativos numericamente.

Destaca-se nesses reservatórios a ocorrência do molusco exótico Corbicula flumínea (Muller, 1774). Trata-se de um molusco originário do Sul da China teve seu 
primeiro registro no Rio Grande do Sul no final da década de 1970 (VEITENHEIMERMENDES, 1981) e tem sido registrado em ambientes aquáticos das regiões sul e sudeste e também na Bacia Amazônica. Além da competição com as espécies nativas esse molusco pode, em grandes quantidades, obstruir a passagem de água tanto em usinas hidrelétricas quanto nos canais de abastecimento nesses locais (MANSUR \& GARCES, 1988).

De maneira geral, as águas dos reservatórios são neutras, condutividade elétrica relativamente baixa e bem oxigenada (Tabela 5).

Tabela 2. Composição taxonômica, abundância absoluta e relativa (\%) e densidade numérica (ind. $\mathrm{m}^{2}$ ) dos macroinvertebrados bentônicos no reservatório de Jaguari em agosto de 2012.

\begin{tabular}{cccc|c|c|c|c|c}
\hline Classe/Ordem & Família & JA 01 & JA 02 & JA 03 & AA & AB\% & Dens $\mathbf{~ m}^{2}$ \\
\hline Diptera & Chironomidae & 36 & 3 & 27 & 66 & 25,48 & 461,22 \\
& Ceratopogonidae & 1 & 0 & 0 & 1 & 0,39 & 6,99 \\
& Chaoboridae & 0 & 0 & 2 & 2 & 0,77 & 13,98 \\
Odonata & Libellulidae & 1 & 0 & 0 & 1 & 0,39 & 6,99 \\
Oligochaeta & & 154 & 3 & 31 & 188 & 72,59 & 1313,77 \\
Bivalve & Corbiculidae & 1 & 0 & 0 & 1 & 0,39 & 6,99 \\
\hline Total & & $\mathbf{1 9 3}$ & $\mathbf{6}$ & $\mathbf{6 0}$ & $\mathbf{2 5 9}$ & $\mathbf{1 0 0}$ & $\mathbf{1 8 0 9 , 9 2}$ \\
\hline Riqueza de táxons & & $\mathbf{5}$ & $\mathbf{2}$ & $\mathbf{3}$ & - & - & - \\
\hline
\end{tabular}


Tabela 3. Composição taxonômica, abundância absoluta e relativa (\%) e densidade numérica (ind. $\mathrm{m}^{2}$ ) dos macroinvertebrados bentônicos no reservatório de Jacareí em agosto de 2012.

\begin{tabular}{ccccc|c|c|c|c|c}
\hline Classe/Ordem & Família & JC 01 & JC 02 & JC 03 & JC 04 & AA & AB\% & Dens $\mathbf{~ m}^{2}$ \\
\hline Diptera & Chironomidae & 30 & 273 & 5 & 41 & 349 & 77,56 & 2438,85 \\
& Ceratopogonidae & 0 & 1 & 0 & 0 & 1 & 0,22 & 6,99 \\
& Chaoboridae & 0 & 1 & 31 & 5 & 37 & 8,22 & 258,56 \\
& Tipulidae & 0 & 2 & 0 & 0 & 2 & 0,44 & 13,98 \\
Ephemeroptera & Leptophlebiidae & 0 & 0 & 0 & 1 & 1 & 0,22 & 6,99 \\
Oligochaeta & & 35 & 0 & 0 & 13 & 48 & 10,67 & 335,43 \\
Bivalve & Corbiculidae & 11 & 0 & 0 & 0 & 11 & 2,44 & 76,87 \\
Pupa (diptera) & & 0 & 1 & 0 & 0 & 1 & 0,22 & 6,99 \\
\hline Total & & $\mathbf{7 6}$ & $\mathbf{2 7 8}$ & $\mathbf{3 6}$ & $\mathbf{6 0}$ & $\mathbf{4 5 0}$ & $\mathbf{1 0 0}$ & $\mathbf{3 1 4 4 , 6 5}$ \\
\hline Riqueza de táxons & & $\mathbf{3}$ & $\mathbf{5}$ & $\mathbf{2}$ & $\mathbf{4}$ & - & - & - \\
\hline
\end{tabular}

A Figura 2 mostra a similaridade da composição dos macroinvertebrados bentônicos entre os pontos dos reservatórios Jaquari (JA) e Jacareí (JC). Esta análise evidenciou similaridade da composição da fauna bentônica entre os pontos, JA 02 e JC 01 e entre os pontos JA 04 e JC 04. 


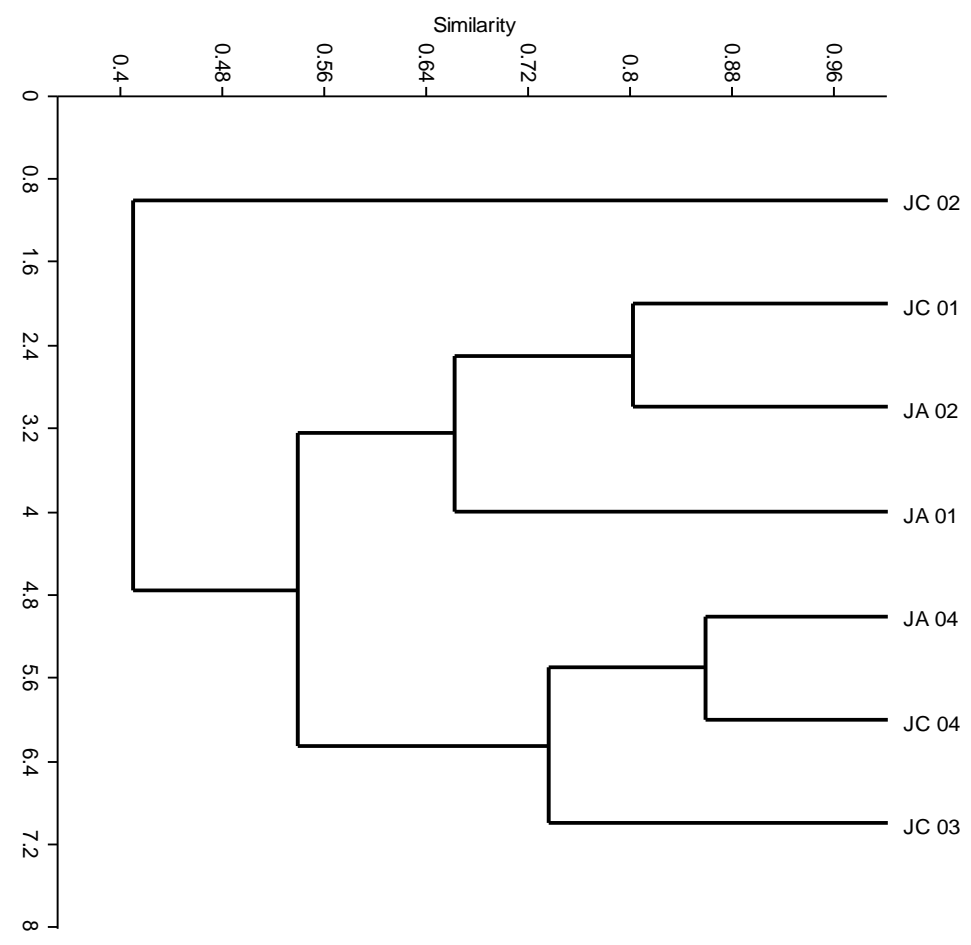

Figura 2. Índice de similaridade entre os reservatórios de Jaguari e Jacareí em agosto de 2012.

Tabela 4. Valores do IET (P) e classificação trófica nos pontos dos reservatórios de Jaguari (JA) e Jacareí (JC) em agosto de 2012.

\begin{tabular}{ccc}
\hline Ponto & IET (Toledo Jr) & Classificação Trófica \\
\hline JA 01 & 54,93 & Eutrófico \\
JA 02 & 36,50 & Oligotrófico \\
JA 03 & 41,91 & Oligotrófico \\
JC 01 & 40,40 & Oligotrófico \\
JC 02 & 50,48 & Mesotrófico \\
JC 03 & 37,64 & Oligotrófico \\
JC 04 & 50,82 & Mesotrófico \\
\hline
\end{tabular}


Tabela 5. Valores médios de $\mathrm{pH}$, condutividade elétrica $(\mathrm{uS} / \mathrm{cm})$, oxigênio dissolvido ( $\mathrm{mgL}$ ) e temperatura da água nos pontos dos reservatórios de Jaguari (JA) e Jacareí (JC) em agosto de 2012.

\begin{tabular}{c|c|c|c|c}
\hline & $\mathbf{p H}$ & Condutividade elétrica $\mathbf{\mu S}-\mathbf{1}^{-}$ & OD $\mathbf{~ m g} / \mathbf{L}$ & Temp ${ }^{\circ} \mathbf{C}$ \\
\hline JA 01 & 7,1 & 30,4 & 8,8 & 16,4 \\
JA 02 & 6,4 & 27,4 & 7,4 & 18,7 \\
JA 03 & 6,4 & 28,1 & 8,3 & 18,6 \\
JC 01 & 6,3 & 27 & 8,94 & 19,8 \\
JC 02 & 6,9 & 27,3 & 8,5 & 19,6 \\
JC 03 & 7,0 & 28,0 & 8,5 & 19,4 \\
JC 04 & 6,7 & 27,9 & 7,7 & 19,1 \\
\hline
\end{tabular}

\section{CONCLUSÃO}

- Oligochaeta foi o táxon numericamente dominante no reservatório de Jaguari e Chironomidae no reservatório de Jacareí;

- Foi registrada a ocorrência do molusco exótico Corbicula flumínea (Muller, 1774) nos pontos JA 01 (Jaguari) e JC 01 (Jacareí) sendo essa espécie causadora de impactos tanto na estrutura da composição dos macroinvertebrados bentônicos, bem como nos canais das usinas hidrelétricas e de abastecimento;

- A elevada abundância registrada de Oligochaeta no ponto JA 01 provavelmente está relacionado ao elevado grau de trofia determinado nesse local.

- A análise de similaridade evidenciou maior similaridade da composição da fauna bentônica entre os pontos, JA 02 e JC 01 e entre os pontos JA 04 e JC 04.

- As águas dos dois reservatórios podem ser consideradas neutras $(\mathrm{pH})$ e bem oxigenadas em todos os pontos de amostragem. 


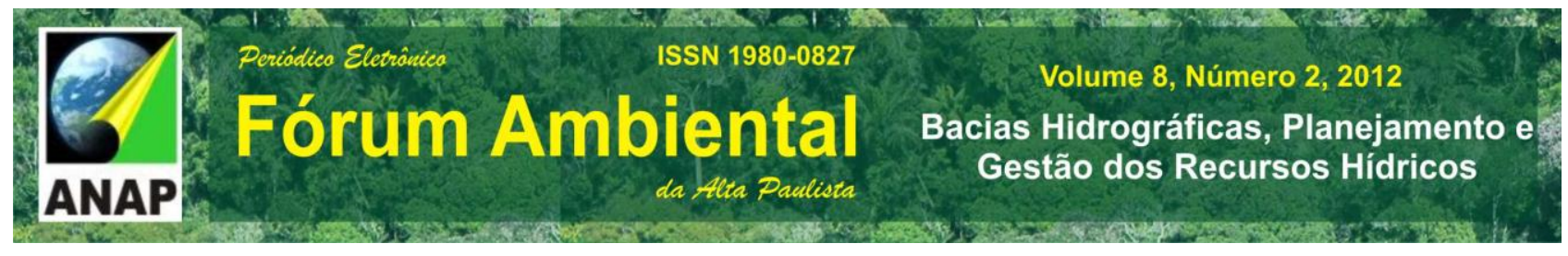

\section{REFERÊNCIAS}

BRINKHURST, R. O. \& MARCHESE, M. R. Guia de la identificacion de Oligoquetos acuaticos continentales de Sud y Centroamerica. Associación Ciencias Naturales del Litoral. Argentina. Colección Climax (5), 207p, 1989.

BRINKHURST R.O, JAMIESON B.G.M. Aquatic Oligochaeta of the World, Edinburgh: Oliver \& Boyd, pp 1-860, 1971.

CALLISTO, M. et al. Aplicação de um protocolo de avaliação rápida de diversidade de habitats em atividades de ensino e pesquisa (MG-RJ). Acta Limnológica Brasiliensis, v. 34, p. 91-98, 2002.

CRANSTON, P. S. Introduction to the Chironomidae. In: P. ARMITAGE, P. S. CRANSTON \& C. V. PINDER (eds.), The Chironomidae: the biology and ecology of non-biting midges. Chapman \& Hall, New York, p. 1-7, 1995.

DI GIOVANNI, M.V. GORETTI, E. \& TAMANTI, V. Macrobenthos in Montedoglio Reservoir, Central Italy. Hidrobiologia, 321: 17 - 28, 1996

EPLER, JH. Identification manual for the larval Chironomidae (Diptera) of Florida. Florida: Department of Environmental Regulation. 427 p, 1992.

HAMMER, O.; HARPER, D. A. T. \& RIAN, P. D. Past: Palaeonthological statistics software package for education and data analysis. Version. 1.37,2001.

LUCCA, J.V. Composição, distribuição e abundância dos macroinvertebrados bentônicos da Lagoa do Caçó (MA). Dissertação de mestrado. Escola de Engenharia de São Carlos - Universidade de São Paulo, 2002.

MANSUR, M.C.D \& GARCES, M.M.P. Ocorrência e densidade de Corbicula fluminea ( Muller, 1774) e Neocorbicula limosa ( Maton 1811) na Estação Ecológica do Taim e áreas adjacentes, Rio Grande do Sul, Brasil. Iheringia. Sér Zoo, Porto Alegre (69) 99116, 1988.

MARGURRAN, A. E. Ecological diversity and its measurement. Princeton, Princeton University, 179p, 1988.

MERRITT, R. W. \& CUMMINS, K. W. An introduction to aquatic insects of North America. Kendall/Hunt Publ. Co. 826p,1996.

PINDER, L.C.V. The larvae of Chironomidae (Díptera) of the Holartic region- Introduction. Entomologica Scandinavica Supplement, v. 19, p. 7-10, 1983. 
RIGHI, G. Manual de identificação de invertebrados Límnicos do Brasil. Brasília, CNPq, 1984.

ROLDAN-PÉRES, G. Guía para el estudo de los macroinvertebrados acuáticos del Departamento de Antioquia. Bogotá: Universidade de Antioquia. 217 p, 1988.

ROSEMBERG, D.M. \& RESH, V.H. Introduction to freshwater biomonitoring and benthic macroinvertebrates. Freshwater Biomonitoring and Benthic Macroinvertebrates (eds D.M. Rosenberg \& V.H. Resh). Chapman \& Hall, London. 1-9,1993.

SILVEIRA, M. P. Aplicação do biomonitoramento da qualidade da água em rios. Meio Ambiente. Documentos n. 36, Embrapa, , 68 p, 2004.

SORENSEN, T. A method for establishing groups of equal amplitude in plant sociology based on similarity of species content and its application to analyses of the vegetation on Danish commons. Biologiske Skrifter 5:1-34, 1948.

TRIVINHO-STRIXINO, S \& STRIXINO, G. Larvas de Chironomidae do Estado de São Paulo. Guia de identificação e diagnose dos gêneros. São Carlos: PPG-ERN/UFSCar, 229p, 1995.

TOLEDO Jr., AP., TALARICO, M., CHINEZ, SJ. and AGUDO, EG. A aplicação de modelos simplificados para a avaliação do processo da eutrofização em lagos e reservatórios tropicais. In Anais do 12 Congresso Brasileiro de Engenharia Sanitária e Ambiental. Camboriú: DENG,1983.

VALDERRAMA, J.C. The simultaneous analisys of total nitrogen and phosphorus in natural waters. Mar. Chem., v.10, p.109-122,1981.

VEITENHEIMER,M. Corbicula manilensis (Philippi, 1844) molusco asiático, na bacia do Jacuí e do Guaiba, Rio Grande do Sul, Brasil (Bivalvia, Corbiculidae). Iheringia, Série Zoa!. (60):63-74, 1981.

WELCH, W. H. Mosses and their uses. Proc. Indiana Acad. Sci,1948. 